\title{
Excitation Mechanism in roAp Stars
}

\author{
N. J. Balmforth ${ }^{1}$, M. S. Cunha ${ }^{2}$, N. Dolez ${ }^{4}$, D. O. Gough ${ }^{2,3}$, S. Vauclair ${ }^{4}$
}

\begin{abstract}
In the present work we develop a theoretical model for roAp stars characterized by the suppression of convection around the magnetic poles. When calculating the growth rates of acoustic oscillations in models of this type we find that most models whose positions in the HR diagram coincide with that of the observed roAp stars are unstable against high-order pulsations.
\end{abstract}

\section{Introduction}

The magnetic field in roAp stars might be strong enough to suppress turbulent motion near the magnetic poles. Accordingly, and following the work by Dolez \& Gough (1982), we develop a model composed of two regions: a polar region, where convection is totally suppressed, and an equatorial region, where the latter is left untouched. Moreover, the helium abundance distributions in the equatorial and polar regions are chosen such as to be in agreement with the time-dependent diffusion results of Vauclair, Dolez \& Gough (1991).

The growth rates of the acoustic oscillations are calculated with envelope models similar to those described by Baker \& Gough (1979), and developed further by Balmforth (1992) and Houdek (1996). For each star, two spherically symmetric models are built, one with the characteristics of the equatorial region and the other with the characteristics of the polar region. The growth rates of radial oscillations of the composite model contain contributions from these two regions and are obtained by carrying out an appropriately weighted average of the growth rates of each of the two spherical models (see Cunha 1999).

\section{Results}

The results obtained for ten theoretical models, chosen so as to cover the region of the HR diagram where the observed roAp stars are believed to be positioned, show that high order pulsations might be excited around the magnetic poles

\footnotetext{
${ }^{1}$ Department of Applied Mathematics and Statistics, Jack Baskin School of Engineering, 139 Baskin Engineering Bldg, University of California, Santa Cruz, CA 95064, USA

${ }^{2}$ Institute of Astronomy, Madingley Road, Cambridge CB3 0HA, England

${ }^{3}$ Department of Applied Mathematics and Theoretical Physics, University of Cambridge, U.K.

${ }^{4}$ Observatoire Midi-Pyrénées, 14 Avenue Edouard Belin, F-31400 Toulouse, France
} 


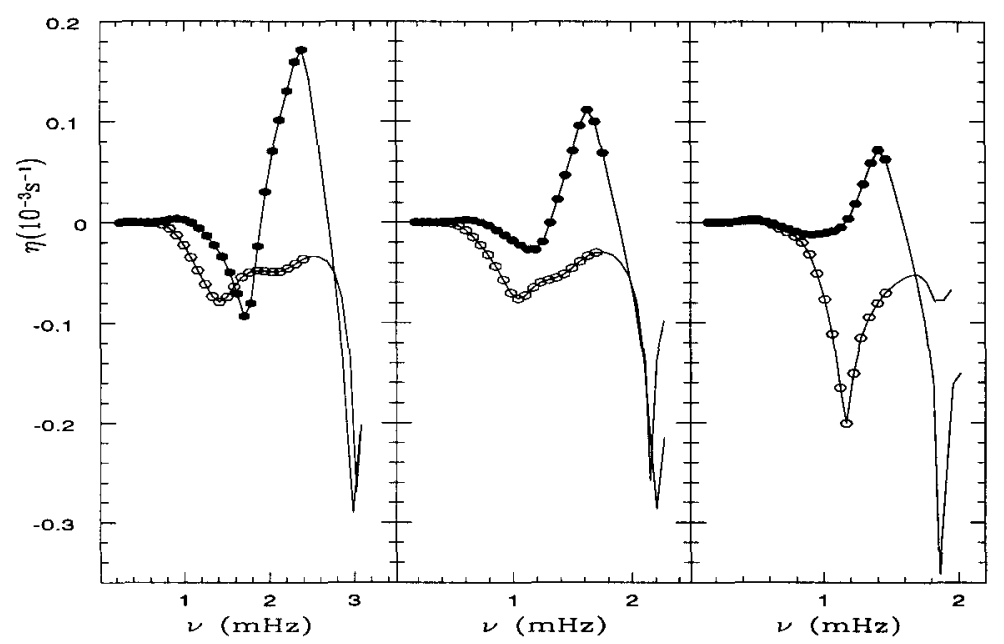

Figure 1. Growth rates of radial modes of three theoretical spherical models of mass $M=1.87 M_{\odot}$, as a function of frequency $\nu$. Full circles show the results for the polar regions of our composite Ap star, open circles for the equatorial regions. The effective temperatures and luminosities of the models are, from left to right, $\left(\log T_{\text {eff }}=3.936\right.$, $\left.\log L / L_{\odot}=1.086\right),\left(\log T_{\text {eff }}=3.910, \log L / L_{\odot}=1.164\right)$ and $\left(\log T_{\text {eff }}=\right.$ $\left.3.893, \log L / L_{\odot}=1.187\right)$.

of these stars. In most cases the contribution from the polar regions are sufficiently large to overcome the negative contribution from the equatorial regions, rendering the high-order modes unstable. Moreover, it is clear from the results that these modes are excited by the $\kappa$-mechanism acting in the region of partial hydrogen ionization, as has already been suggested by Dziembowski \& Goode (1996). Fig. 1 shows the growth rates obtained from the polar and equatorial regions of three of our models.

\section{References}

Baker, N. H. \& Gough, D. O. 1979, ApJ, 234, 232

Balmforth, N. J. 1992, MNRAS, 255, 603

Cunha, M. S. 1999, PhD thesis, Cambridge, U.K.

Dolez, N. \& Gough, D. O. 1982, in Pulsations in Classical and Cataclysmic Variable stars, ed. J. P. Cox \& C. J. Hansen (Boulder: JILA), 248

Dziembowski, W. A. \& Goode, P. R. 1996, ApJ, 458, 338

Houdek, G. 1996, PhD Thesis, Vienna, Austria

Vauclair, S., Dolez, N., \& Gough, D. 1991, A\&A, 252, 618 